\title{
Direct UV photocrosslinking of poly( $N$-vinyl-2-pyrrolidone) (PVP) to produce hydrogels
}

\author{
Lilian C. Lopérgolo ${ }^{\mathrm{a}, \mathrm{b}}$, Ademar B. Lugão ${ }^{\mathrm{b}}$, Luiz H. Catalani ${ }^{\mathrm{a}, *}$ \\ anstituto de Química, Universidade de São Paulo, CP 26.077, 05513-970, São Paulo-SP, Brazil \\ ${ }^{\mathrm{b}}$ Instituto de Pesquisas Energéticas e Nucleares IPEN-CNEN/SP-Travessa R, 400, 05508-970, São Paulo-SP, Brazil
}

Received 13 May 2003; received in revised form 24 July 2003; accepted 25 July 2003

\begin{abstract}
Hydrogels for biomedical purposes, made from synthetic polymers as starting materials and free of co-adjuvant molecules, have been produced almost exclusively by high-energy radiative processes. On the other hand, UV photocrosslinking of such materials has been used in conjunction of monomers and/or photoinitiators. This work was addressed to the analysis of poly( $N$-vinyl-2-pyrrolidone) (PVP) submitted to direct photocrosslinking in aqueous solution, using low pressure $\mathrm{Hg}$ lamp $\left(\lambda_{\mathrm{em}}=254 \mathrm{~nm}\right)$. The process efficiency was evaluated, and the properties of the hydrogel formed were determined. The product thus formed has similar micro- and macroscopic properties, as compared to hydrogels produced by high-energy radiation and presents no cytotoxicity. These results demonstrated the viability of using this method as a versatile alternative to hydrogel production, broadening the possibility of its production where high-energy radiation facilities are not available.

(C) 2003 Published by Elsevier Ltd.
\end{abstract}

Keywords: Poly(N-vinyl-2-pyrrolidone); Hydrogel; Photocrosslinking

\section{Introduction}

The use of hydrogels as biomaterials has recently gained great importance in view of the low toxicity and high biocompatibility presented by many of them $[1,2]$. The main areas of hydrogels applications today include [3]: (i) topical applications as wound dressings; (ii) drug delivery systems; (iii) transdermal systems; (iv) dental applications; (v) injectable polymers; (vi) implants; (vii) ophthalmic applications and (viii) stimuli-responsive systems.

Permanent hydrogels, as opposed to reversible hydrogels, consist of covalently crosslinked hydrophilic polymers that may absorb water several thousand times of their dry weight without being dissolved. They can be produced either by in situ monomer polymerization in the presence of a bifunctional monomer, or by crosslinking the preformed macromolecule by any convenient method. For biomedical applications, the second type is preferred since no residual monomer is left as a potential toxic agent.

\footnotetext{
* Corresponding author. Address: Institute of Chemistry, University of São Paulo, Av. Prof. Lineu Prestes, 748, 05508900, São Paulo, Brazil. Tel.: +55-11-3091-3162; fax: +55-11-3815-5579.

E-mail address: catalani@iq.usp.br (L.H. Catalani).
}

Photopolymerizable hydrogels have been proposed for a number of different biomedical applications, like drug delivery systems, cell encapsulation, scaffold materials and as a barrier in tissue injury [4]. In this case, a pre-gel liquid solution could be delivered by a non-invasive technique, and crosslinking can be accomplished in situ provided the method is fast. The feasible methods are, however, always dependent on photoinitiators acting upon macromonomers (a macromolecule derivatized into a vinylic monomer). The presence of vinylic residues, initiators and their subproducts may render unsuitable toxic properties to these materials.

Rosiak and coworkers [5,6] have presented a successful methodology of hydrogel dressing production based on high-energy radiation, like electron beam or gamma radiation. Their basic composition is poly( $N$-vinyl-2pyrrolidone) (PVP), poly(ethyleneglycol) (PEG), agar and water. In the end-use form, they represent transparent sheets of the thickness of a few millimeters, containing over $90 \%$ of water. These dressings have been commercialized as wound dressing under the trade name $\mathrm{HDR}^{\circledR}$ and AQUAGEL $^{\circledR}$. However, they have also been used for medical treatment of other types of wounds and illnesses.

The primary process involved in Rosiak's method is 
water radiolysis. Hydroxyl radicals thus formed react with PVP generating macroradicals. One of the resulting reactions from these radicals is recombination, leading to crosslinking. Restrictions of this method are two-fold: (i) the need of special facilities for the high energy sources and (ii) the potent degenerative power of the radiation used, precluding the combined use of more sensitive material like proteins and any other biological material.

Ultraviolet radiation may represent an option against high-energy radiation. Since PVP has an absorption spectrum in the region of $200-280 \mathrm{~nm}$, the use of arc mercury lamps seems plausible. The photochemistry of the pyrrolidone substituent, a cyclic amide, may lead to radical formation, the primary requirement for reticulation.

In this work, we present an alternative methodology based on the use of ultraviolet radiation. The crosslinking process was followed and its product investigated.

\section{Methods}

\subsection{Materials}

Poly( $N$-vinylpyrrolidone) known as Plasdone K-90 $\left(\bar{M}_{\mathrm{w}}=1.2 \times 10^{6} ; \bar{M}_{\mathrm{n}}=3.6 \times 10^{5}\right)$ was from GAF Chem. Co. (USA).

\subsection{Irradiation of PVP solution}

Irradiations were carried out using a PCQ-X1 reactor from Ultra Violet Products (Cambridge, UK) with 3 lowpressure doughnut-type $\mathrm{Hg}$ lamps of $17.2 \mathrm{~W} / \mathrm{lamp}$ $\left(\lambda_{\mathrm{em}}=254 \mathrm{~nm}\right)$ and $7.5 \mathrm{~cm}$ of diameter. A PVP $80 \mathrm{mg} / \mathrm{ml}$ solution was placed in a $1 \mathrm{~cm}$ quartz tube placed in the center of the lamp set. This setting produces $3.6 \mathrm{MW} / \mathrm{cm}^{2}$ of radiant flux at its center. The rod shaped specimens were used in this setting $(\phi=1 \mathrm{~cm} ; h=10 \mathrm{~cm})$ receives $113 \mathrm{~mJ} / \mathrm{s}$. Alternatively, the solution was placed in a flat quartz mold $(\ell=170 \mathrm{~cm} ; w=2.5 \mathrm{~cm} ; h=0.3 \mathrm{~cm})$ and a pen-type Heraeus (Hanau, Germany) low-pressure Hg lamp $200 \mathrm{~mm}$ long of $20 \mathrm{~W}$ was placed longwise $20 \mathrm{~mm}$ distant from the top of the solution. This setting produces 11.5 $\mathrm{MW} / \mathrm{cm}^{2}$ of radiant flux at the quartz mold position, or $4.89 \mathrm{~J} / \mathrm{s}$ on the flat specimen. The radiance measurements were dome using a Solar Light Radiometer (Philadelphia, USA), Model PMA 2200, with a UV detector Model PMA 2122.

\subsection{Mechanical tests}

The mechanical properties were determined using an Instron Model 5567 testing instrument at a strain rate of $25 \mathrm{~mm} / \mathrm{min}$. The samples were prepared according to ABNT NBR 6241/80.

\subsection{Gel content of PVP hydrogel}

PVP membranes were subjected to Soxhlet extraction with water for $36 \mathrm{~h}$ to remove sol content and then dried under vacuum at $60{ }^{\circ} \mathrm{C}$ for $24 \mathrm{~h}$ to a constant weight. The gel content was calculated as follows:

$s(\%)=\left(\frac{w_{\mathrm{g}}-w_{0}}{w_{0}}\right) 100$

$g(\%)=100-s(\%)$,

where $s(\%)=$ sol content; $g(\%)=$ gel content; $w_{\mathrm{g}}=$ weight of PVP used to prepare hydrogel before irradiation; $w_{0}=$ weight of dried gel.

\subsection{Crosslinking density and mesh size determination}

The crosslinking density $\rho_{x}$ was calculated as the reciprocal of $\bar{v} M_{\mathrm{c}}$, the product of specific volume and the number-average molecular weight between crosslinks, according to Eq. (3) [7].

$$
\begin{aligned}
\rho_{x}=\frac{1}{\bar{v} M_{\mathrm{c}}}= & \frac{2}{\bar{v} \bar{M}_{\mathrm{n}}} \\
& +\frac{\left(1 / V_{1}\right)\left[\ln \left(1-V_{2 \mathrm{~s}}\right)+V_{2 \mathrm{~s}}+\chi_{1} V_{2 \mathrm{~s}}^{2}\right]}{V_{2 \mathrm{r}}\left[\left(V_{2 \mathrm{~s}} / V_{2 \mathrm{r}}\right)^{1 / 3}-1 / 2\left(V_{2 \mathrm{~s}} / V_{2 \mathrm{r}}\right)\right]},
\end{aligned}
$$

where $\bar{M}_{\mathrm{n}}$ is the number-average molecular mass $\left(3.6 \times 10^{5} \mathrm{~g} / \mathrm{mol}\right.$ for the PVP used), $V_{2 \mathrm{~s}}$ is the polymer volume fraction in the swollen gel at equilibrium, $V_{2 \mathrm{r}}$ is the polymer volume fraction in the relaxed state, $V_{1}$ is the molar volume of the solvent, $\bar{v}$ is the polymer specific volume (the reciprocal of the polymer density; $0.785 \mathrm{~cm}^{3} / \mathrm{g}$ for PVP) and $\chi_{1}$ is the solubility interaction parameter for $\mathrm{PVP}$-water system.

Flory-Huggins theory provides a means for experimental determination of the solubility interaction parameter according to Eq. (4) [8]:

$\chi_{1}=\frac{1}{2}-\left[\frac{A_{2} V_{1}}{\left(V_{2} / \bar{M}_{\mathrm{w}}\right)^{2}}\right]$,

where $A_{2}$ is the second virial coefficient, $V_{2}$ is the molar volume of the polymer and $\bar{M}_{\mathrm{w}}$ is the weight-average molecular mass. The light scattering analysis of the PVP used and water as solvent presented a $\bar{M}_{\mathrm{w}}$ value of $1.14 \times 10^{6} \mathrm{~g} / \mathrm{mol}, A_{2}$ as $3.95 \times 10^{-4} \mathrm{~mol} \mathrm{~cm} / \mathrm{g}^{2}$, which resulted in a $\chi_{1}$ value of 0.489 . The light scattering measurements were performed using a Dawn-F DSP photometer from Wyatt Technology (Santa Barbara, USA).

The mesh size $\xi$ (in $\mathrm{nm}$ ) of the polymer network can be calculated by Eq. (5) [9]:

$$
\xi=0.1 V_{2 \mathrm{~s}}^{-1 / 3}\left(C_{\mathrm{n}} \frac{2 M_{\mathrm{c}}}{M}\right)^{1 / 2} \delta,
$$

where $M$ is the monomeric unit molecular mass, $C_{n}$ is the rigidity coefficient, and $\delta$ is the length of an individual $\mathrm{C}-\mathrm{C}$ 
bond $(0.154 \mathrm{~nm})$. The value of 6.9 [9] for poly(methylmethacrylate) was used as $C_{\mathrm{n}}$.

\subsection{Swelling ratio [7]}

PVP membranes were immersed in distilled water at $25^{\circ} \mathrm{C}$ during $12 \mathrm{~h}$, when equilibrium is reached. Then, they were removed from the water, dried quickly with filter paper to remove excess water and weighed. The swelling ratio $(Q)$ was calculated as follows:

$Q=\frac{V_{\mathrm{s}}}{V_{\mathrm{r}}}$

where $V_{\mathrm{s}}$ is the volume of swollen hydrogel and $V_{\mathrm{r}}$ is the volume of relaxed hydrogel.

\subsection{Cytotoxicity tests}

The cytotoxicity of the membranes was tested by an in vitro cell viability method according to Ciapetti et al. [11]. The test was carried out with dilution of the extracts of hydrogel membranes, in contact with mouse subconjunctive tissue cell culture, from NCTC Clone 929 line (ATCCCCL1). The cells were maintained in Eagle culture medium containing $10 \%$ fetal calf serum, non-essential amino acids and sodium pyruvate. A suspension of $5 \times 10^{4}$ cell $/ \mathrm{ml}$ was prepared, $0.2 \mathrm{ml}$ of this suspension was added to 96 -well microplate and maintained $24 \mathrm{~h}$ at $37^{\circ} \mathrm{C}, 5 \% \mathrm{CO}_{2}$ atmosphere until confluence is achieved. The medium was replaced by $0.2 \mathrm{ml}$ of the hydrogel extracts properly diluted (prepared according to ISO 10993-4 and ISO 10993-5). After $24 \mathrm{~h}$ in the same conditions above, the supernatant was replaced by $0.1 \mathrm{ml}$ of medium containing $50 \mu \mathrm{g}$ of neutral red and incubated for $3 \mathrm{~h}$. The plates were washed with $\mathrm{CaCl}_{2}$ solution in formaldehyde and gently shaken with $0.2 \mathrm{ml}$ of acetic acid/methanol mixture (1:1) for $10 \mathrm{~min}$. The absorbance readings at $540 \mathrm{~nm}$ were obtained from a SLT Spectra spectrophotometer for microplates (SLT Labinstruments, Viena, Austria). Phenol solution (0.02\%) and poly(vinyl chloride) (PVC) extracts were used as positive and negative controls, respectively.

Primary dermal inflammation tests were performed according to Draize et al. [12]. New Zealand male albino rabbits weighing around $2 \mathrm{~kg}$ were used. The dorsal region was trichotomized and divided in two sections, one serving as control. Hydrogel samples were applied, covered with sterile gauze and fixed with hypoallergenic tape. Edema and erythema readings were performed after 24 and $72 \mathrm{~h}$.

\section{Results and discussion}

\subsection{Irradiation of the PVP aqueous solution}

The PVP aqueous solution shows a shoulder of an absorption band at $254 \mathrm{~nm}$, representing a molar extinction coefficient $(\varepsilon)$ equal to $5.15 \mathrm{M}^{-1} \mathrm{~cm}^{-1}$, relative to the repeating unit. For a flat membrane of $80 \mathrm{mg} / \mathrm{ml}$ PVP solution, with an average thickness of $0.3 \mathrm{~cm}, 92.3 \%$ of the radiation that reaches the solution is absorbed. Alternatively, rod shaped hydrogel specimens of $1.0 \mathrm{~cm}$ diameter were produced using the same solution, irradiated on a quartz tubing and using circular lamps. In this case, the UV radiation was $98.6 \%$ absorbed before reaching the rod center and $\sim 100 \%$ before crossing it, demonstrating that photochemical events are possible throughout the initial solution. Indeed, an apparent homogeneous radial consistence is perceptible when $80 \mathrm{mg} / \mathrm{ml}$ solution is used.

Total gel fraction is dependent on the irradiation time. Fig. 1(A) shows the dependence of gel content, $g(\%)$, with increasing radiation dose. In all cases, gel content increases to a maximum and then it levels up. Fig. 1(B) shows the $g(\%)$ at different PVP concentrations after $1 \mathrm{~h} 20 \mathrm{~min}$ of irradiation, which corresponds to a radiation dose of $1.55 \mathrm{~kJ}$. The optimum concentration was found to be ca. 8\% PVP.

If $\mathrm{N}_{2}$ is flown through the irradiation chamber, the gel fraction drops ca. $15-20 \%$, when a dose of $1.55 \mathrm{~kJ}$ is used. Oxygen is clearly an important assistant reagent, enhancing
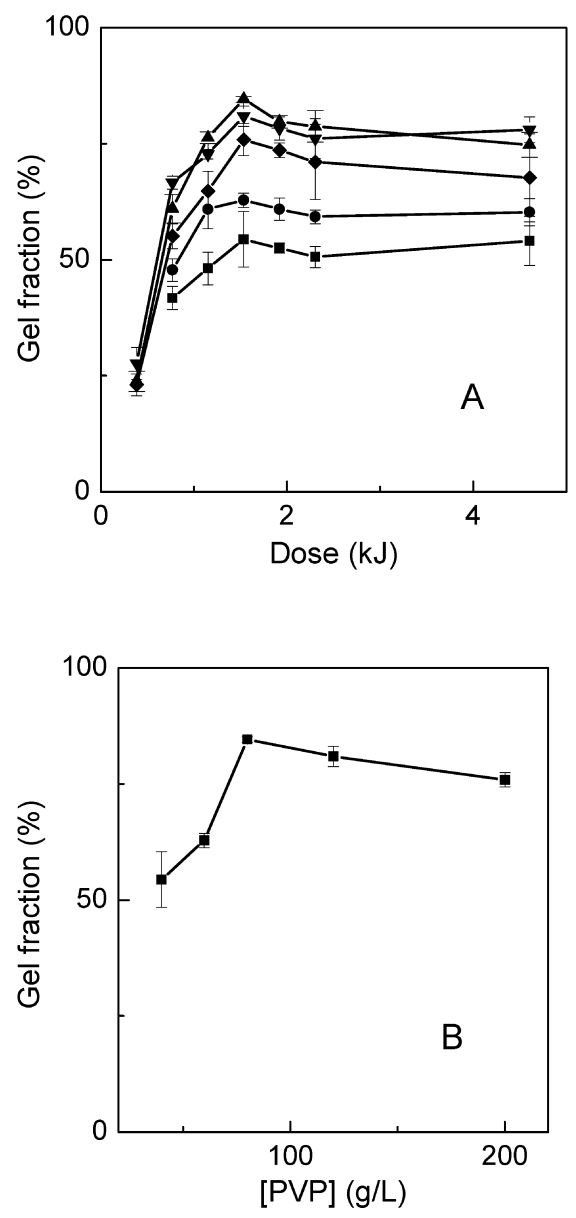

Fig. 1. Effect of irradiation dose and PVP concentration on the gel content. Inset A: (-口-) $40 \mathrm{mg} / \mathrm{ml}$; (-○-) $60 \mathrm{mg} / \mathrm{ml} ; \quad(-\boldsymbol{\Delta}-) \quad 80 \mathrm{mg} / \mathrm{ml} ; \quad(-\nabla-)$ $120 \mathrm{mg} / \mathrm{ml}$; (-) $200 \mathrm{mg} / \mathrm{ml}$. Inset B corresponds to a fixed dose of $1.55 \mathrm{~kJ}$ upon the rod shaped specimens. 
crosslinking, most probably through peroxide formation followed by its decomposition. However, this number also means that direct crosslinking reactions, without the assistance of oxygen, is also a major event responsible for the formation of a $70 \%$ gel fraction in the $\mathrm{N}_{2}$ purged chamber.

Very little is known about photochemistry of 2pyrrolidone moiety. However, basic amide and lactams photoreactions are expected like: $\beta$-cleavage, inter- and intramolecular photoreduction, photorearrangements, etc. Since all these reactions are intermediated by radicals, it is valid to suggest that radicals at the polymer backbone are also formed. The crosslinking takes place by recombination of these radicals. At high concentration, an excimer is formed by photoenolization products [13]. This reaction must be lowering the efficiency of the process, as an alternative deactivation process.

The reason for the leveling of Fig. 1(A) is that, at higher doses the rate of degradation processes is closer to rate of crosslinking. Charlesby and Pinner [14] have proposed a method to access the ratio of degradation to crosslinking density, according to the Eq. (7):

$s+\sqrt{s}=\frac{p_{0}}{q_{0}}+\frac{2}{q_{0} u D}$,

where $s$ is sol fraction, $p_{0}$ is the degradation density (mean value of chain scissions per dose and monomeric unit), $q_{0}$ is the crosslinking density (mean value of crosslinks per dose and monomeric unit), $D$ is the applied radiation dose, and $u$ is the polymerization degree. The linear coefficient of $(s+$ $\sqrt{s})$ vs. $1 / D$ plot is the ratio $p_{0} / q_{0}$.

Fig. 2 shows this analysis for the irradiation of $8 \%$ PVP aqueous solution with doses between 0.38 and $1.92 \mathrm{~kJ}$. The linear regression gave a $p_{0} / q_{0}$ value of $0.25 \pm 0.07$, which means that crosslinking occurs four-fold faster than chain scission, at most. It also shows that for higher doses scattering is high and linearity is lost, pointing to a higher degree of degradation.

Olejniczak et al. [10] presented an explanation for this lack of linearity in other systems. They argue that for

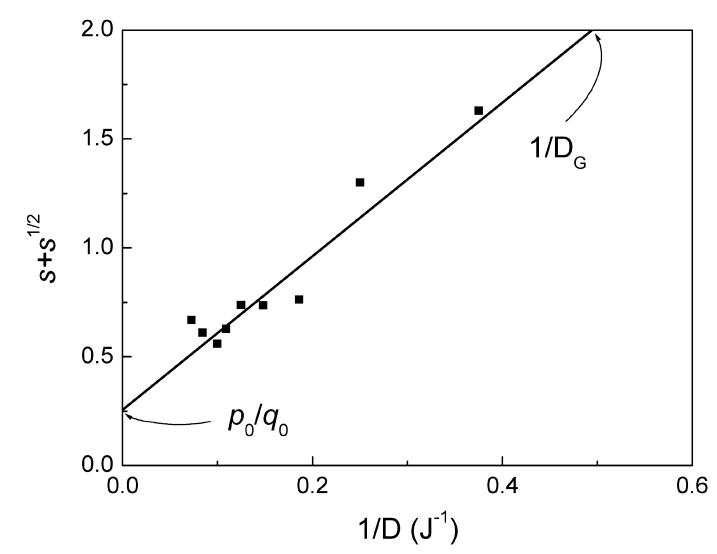

Fig. 2. Sol/gel data treatment according to Charlesby-Pinner relation. $[\mathrm{PVP}]=80 \mathrm{~g} / \mathrm{l}$ in water at room temperature. systems that are far from the original premise of a random molecular weight distribution, a correction factor must be introduced to achieve linearity. By applying CharlesbyRosiak approach we found a $p_{0} / q_{0}$ value of $0.34 \pm 0.06$, not very different from the original value.

In either case, the $p_{0} / q_{0}$ values are high when compared to the literature. Olejniczak et al. [10] reported $p_{0} / q_{0}$ values for $\gamma$-irradiated PVP aqueous solution of 0.22 at normally aerated solution and zero from an Argon saturated solution. The long irradiation periods involved in UV-crosslinking seems to be responsible for this difference. Another possible explanation for this fact is simply that degradation products might be interfering, either by precluding crosslinking or enhancing degradation processes.

This data treatment also provides a means to calculate the gel dose, $D_{\mathrm{g}}$, which is the dose necessary to produce the first insoluble fraction. The non-corrected $D_{\mathrm{g}}$ value obtained from Fig. 2 is $304 \pm 18 \mathrm{~J}$, while the corrected value is $337 \pm 15 \mathrm{~J}$.

As one would expect, the PVP concentration effect is also significant, since crosslinking is a second order process. Figs. 1 and 2 depict concentration dependence of several factors like: gel fraction, swelling ratio, crosslinking density, the number-average molecular weight between crosslinks $M_{\mathrm{c}}$ and mesh size. In all cases, the concentration range of $8-10 \%$ seems to be optimum.

\subsection{Properties of the hydrogel formed}

The crosslinking density $\rho_{x}$ is the primary factor affecting the properties of the membrane. Peppas and Merrill model [7] provides an estimate of $M_{\mathrm{c}}$, calculated from the polymer volume fractions in the swollen gels at equilibrium (see Eq. (3)). As a subproduct, the mesh size $\xi$ (in nm) of the polymer network can be calculated by the Eq. (5) [7,9].

Fig. 3 shows how the swelling ratio, $Q$, number-average molecular weight between crosslinks, $M_{\mathrm{c}}$, the crosslinking density, $\rho_{x}$, and mesh size, $\xi$, varies with radiation dose and PVP concentration. The format of the curves also demonstrates that the yield of crosslinking is not linearly dependent of irradiation time. However, the minimum $M_{\mathrm{c}}$ obtained is around 30,000, showing that the net is reasonable wide open. At the same dose, crosslinking density rises with the concentration, showing that the proximity of the macroradicals formed is important. Alternatively, in the absence of a second macroradical to form a bond, a born radical will decay trough a different path, most likely leading to degradation.

A better parameter to understand the network microscopic morphology is mesh size, which is important in drug delivery and related systems [9]. Fig. 3(D) shows mesh size dependence on dose and PVP concentration. The control of this parameter can be easily achieved by choosing the correct conditions. However, for gels with reasonable mechanical consistence, a minimum of $70 \%$ gel fraction is 

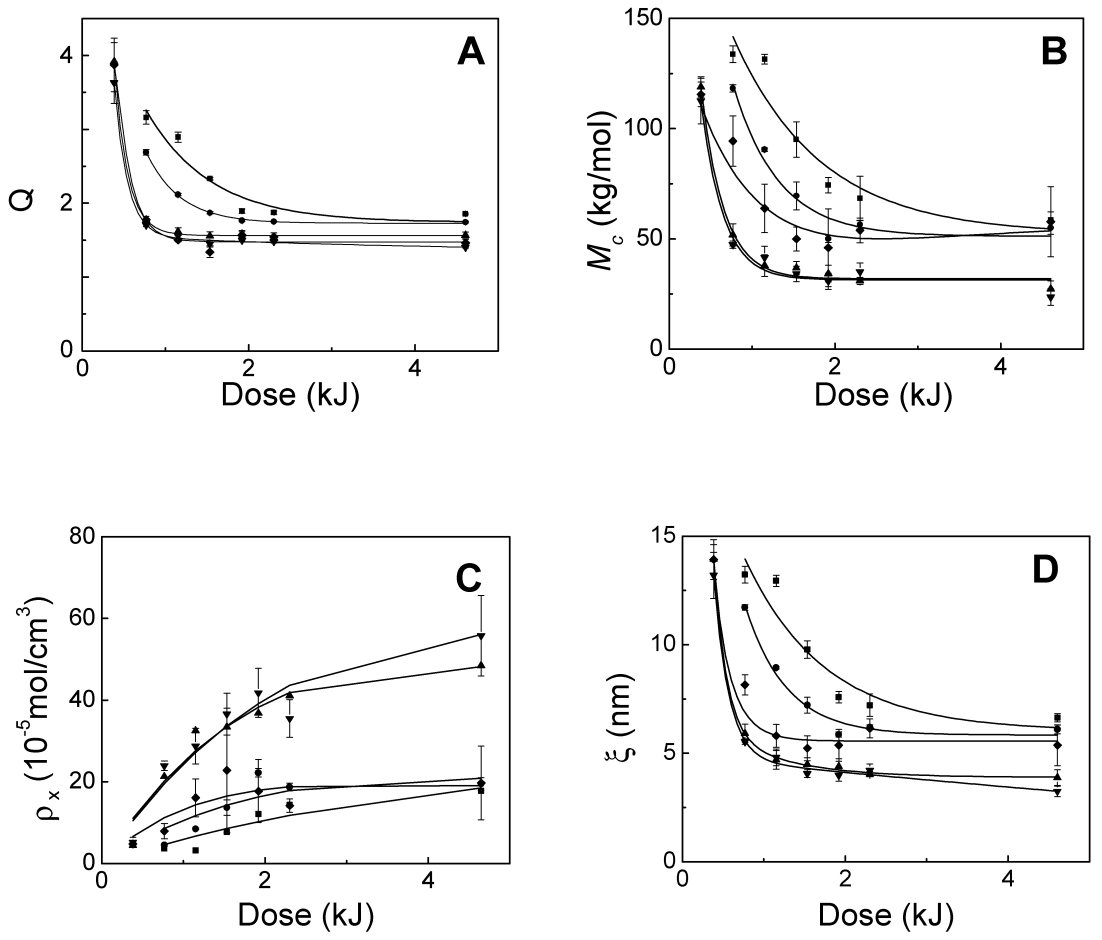

Fig. 3. Effect of irradiation dose and PVP concentration on the (A) swelling ratio at equilibrium, $Q$, (B) the number-average molecular weight between

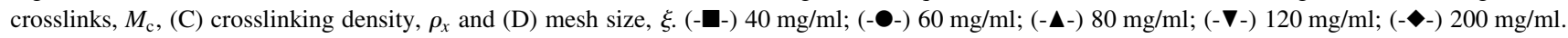

needed (see Fig. 1). Hence, for such cases, mesh size was found to be around $4-6 \mathrm{~nm}$.

The swelling ratio at equilibrium depicts the maximum amount of water the hydrogel can hold, and again this is a function of concentration and dose (Fig. 3(A)). This parameter is especially important in applications where gels are used to absorb exudates, like wound dressings and drug delivery devices. In the last case, the augment of mesh size due swelling is the prime control of delivery rate.

The mechanical properties of the hydrogel were also analyzed. The tensile strength $\left(T_{\mathrm{s}}\right)$ and elongation at break

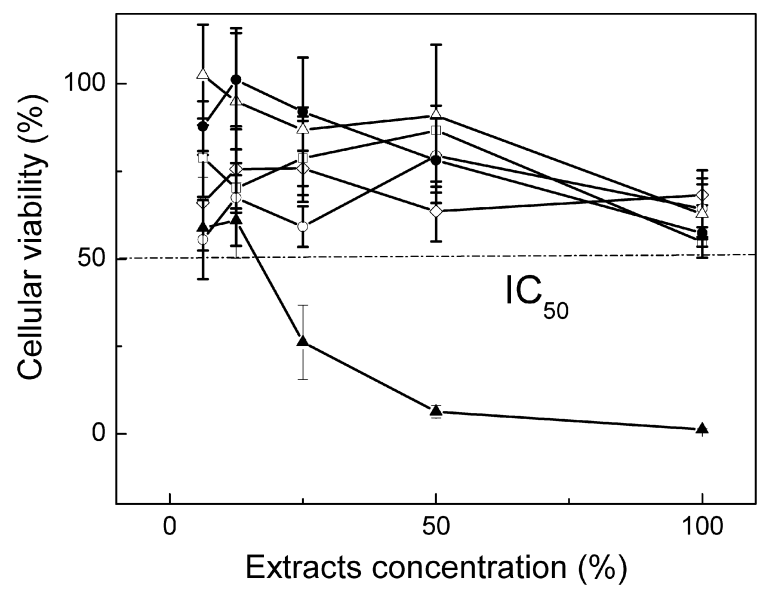

Fig. 4. Colony suppression curves of the hydrogel membranes produced by different UV doses.[PVP] $=80 \mathrm{~g} / \mathrm{l}$ in water at room temperature. $(-\diamond-)$

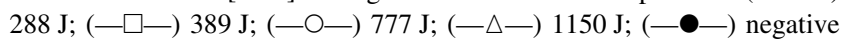
control (PVC); (- - -) positive control (phenol).
$\left(E_{\mathrm{b}}\right)$ were determined for a typical membrane used for dressing (3 mm thick) irradiated with $12.7 \mathrm{~J}$, as a function of PVP concentration. Table 1 lists the values found.

While elongation at break did not present a significant variation, the tensile strength was optimum for PVP concentration of $80-90 \mathrm{mg} / \mathrm{ml}$, decreasing at higher concentration. This can be explained by reviewing Fig. 1(B), where it is clear that the gel fraction decreases at higher concentration, therefore compromising its mechanical properties.

As compared to membranes produced by high-energy radiation, where tensile strength was found to be $0.02 \mathrm{MPa}$ for the same concentration [15], the membrane here reported is ca. $30 \%$ weaker. However, even with this decrease in mechanical quality, the membrane still is quite strong to be handled. Besides, other types of reinforcement may be applied to suite its use [16].

Table 1

Mechanical properties of the hydrogel produced by UV radiation

\begin{tabular}{lllll}
\hline $\begin{array}{l}\text { PVP conc. } \\
(\mathrm{mg} / \mathrm{ml})\end{array}$ & $g(\%)$ & $\begin{array}{l}\rho_{x} \\
\left(10^{-5} \mathrm{~mol} / \mathrm{cm}^{3}\right)\end{array}$ & \multicolumn{1}{l}{$\begin{array}{l}T_{\mathrm{s}} \\
\left(10^{-3} \mathrm{MPa}\right)\end{array}$} & \multicolumn{1}{l}{$\begin{array}{l}E_{\mathrm{b}} \\
(\%)\end{array}$} \\
\hline 80 & $84.5 \pm 0.6$ & $3.47 \pm 3.4$ & $12.3 \pm 2.1$ & $54.5 \pm 5.1$ \\
90 & - & - & $13.0 \pm 1.0$ & $55.3 \pm 1.5$ \\
100 & - & - & $11.6 \pm 1.0$ & $60.0 \pm 5.0$ \\
120 & $80.9 \pm 2.1$ & $3.36 \pm 5.3$ & $10.2 \pm 1.2$ & $62.0 \pm 6.9$ \\
200 & $75.9 \pm 3.4$ & $2.59 \pm 5.1$ & $9.6 \pm 0.9$ & $61.9 \pm 5.0$ \\
\hline
\end{tabular}

Irradiation made on a quartz mold by pen-type lamp; irradiation dose $1.8 \mathrm{~kJ}$. 


\subsection{Cytotoxicity tests}

The cytotoxicity of the hydrogels prepared by all methods was tested according to Ciapetti et al. [11]. The relative count of total viable cells was correlated against hydrogel extracts. For comparison, phenol was used as a positive control and poly(vinyl chloride) as a negative control (Fig. 4). The conclusion from this experiment is that the hydrogels prepared by this method do not impose any toxic effect to live organisms.

These hydrogels were also submitted to dermal inflammation test [12] in albino rabbits, where dressings made of hydrogel were maintained in direct contact with the rabbit skin for $72 \mathrm{~h}$. All samples presented inflammation indexes within a satisfactory range, i.e. as a non-irritating material.

\section{Conclusions}

Photocrosslinking of PVP was confirmed as an inexpensive alternative for PVP hydrogel production. This can be achieved by direct irradiation using a low-pressure Hg lamp eliciting $254 \mathrm{~nm}$ light. The material formed showed to be well suited to all PVP hydrogel applications like wound dressings and drug delivery devices. The easy of its production also opens the possibility of its use in situations where high-energy radiation is not available.

\section{Acknowledgements}

The authors would like to thank Dr Sizue O. Rogero and the Instituto Adolfo Lutz for their contribution in the cytotoxicity tests, the Fundação de Amparo a Pesquisa do Estado de São Paulo, FAPESP, São Paulo, Brazil, and the Conselho Nacional de Desenvolvimento Científico e Tecnológico, CNPq, Brasília, Brazil, for financial support.

\section{References}

[1] Lee KY, Mooney DJ. Chem Rev 2001;101:1869-79.

[2] Hoffman AS. Adv Drug Deliv Rev 2002;43:3-12.

[3] Rosiak JM, Yoshii F. Nucl Instr Methods Phys Res B 1999;151: 56-64.

[4] Nguyen KT, West JL. Biomaterials 2002;23:4307-14.

[5] Rosiak J, Olejniaczak J, Pekala W. Radiat Phys Chem 1990;36: $747-55$.

[6] Rosiak JM, Rucinska-Rybus A, Pekala W. US Patent No 4,871,490, 1989.

[7] Peppas NA, Barr-Howell BD. In: Peppas NA, editor. Characterization of the cross-linked structure of hydrogels. Hydrogels in medicine and pharmacy, vol. 1. Boca Raton: CRC Press; 1986. p. 27-56.

[8] Hiemenz PC, Rajagopalan R. Principles of colloid and surface chemistry, 3rd ed. New York: Marcel Dekker; 1997.

[9] Rosiak JM, Olejniczak J. Radiat Phys Chem 1993;42:903-6.

[10] Olejniczak J, Rosiak J, Charlesby A. Radiat Phys Chem 1991;37: 499-504.

[11] Ciapetti G, Granchi D, Verri E, Savarino L, Cavedagna D, Pizzoferrato A. Biomaterials 1996;17:1259-64.

[12] Draize JH, Wooddard G, Calvery H. J Pharmacol Exp Ther 1944;83: 377-90.

[13] Sarkar A, Chakravorti S. J Chim Phys 1996;93:1281-91.

[14] Charlesby A, Pinner J. Atomic radiation and polymer. New York: Pergamon Press; 1960.

[15] Miranda LF. In: Estudo de parametros de processo para a sintese de membranes hidrofilicas a base de poli( $N$-vinil-2-pirrolidona). PhD Thesis, University of Sao Paulo, 1999.

[16] Lopergolo LC, Lugao AB, Catalani LH. J Appl Polym Sci 2002;86: $662-6$ 